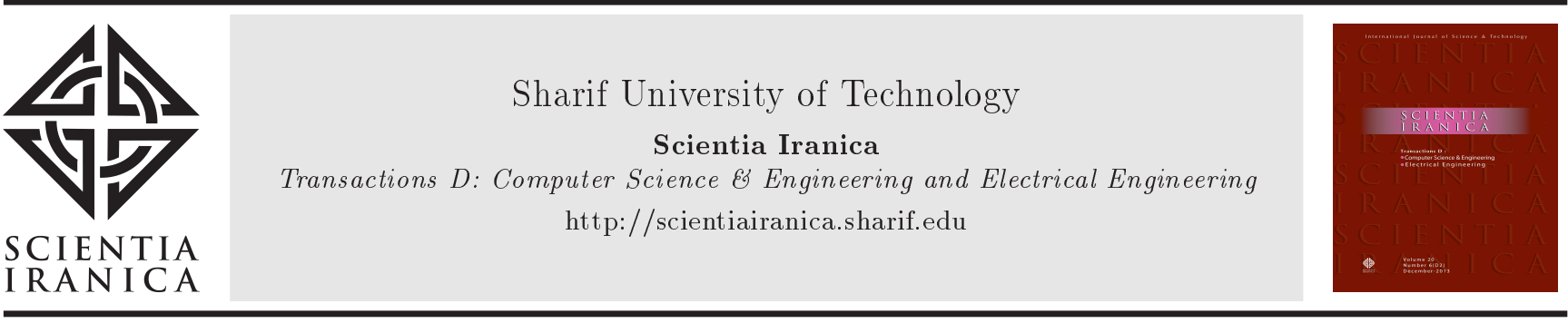

\title{
Portfolio optimization in electricity market using a novel risk based decision making approach
}

\section{S. Bazmohammadi, A. Akbari Foroud*, and N. Bazmohammadi}

Faculty of Electrical \& Computer Engineering, Semnan University, Semnan, Iran.

Received 9 January 2016; received in revised form 3 January 2017; accepted 6 May 2017

\author{
KEYWORDS \\ Decision making \\ approach; \\ Fuzzy satisfaction \\ theorem; \\ Portfolio optimization; \\ Risk management; \\ Stochastic \\ programming.
}

\begin{abstract}
This paper provides generation companies (GENCOs) with a novel decisionmaking tool that accounts for both long-term and short-term risk aversion preferences and devises optimal strategies to participate in energy and ancillary services markets and forward contracts, in which the possibility of involvement in arbitrage opportunities is also considered. Because of the imprecise nature of the decision maker's judgment, appropriate modelling of risk aversion attitude of the GENCO is another challenge. This paper uses fuzzy satisfaction theory to express decision maker's attitude toward risk. Conditional Value at Risk methodology (CVaR) is utilized as the measure of risk and uncertainty sources include prices for the day-ahead energy market, Automatic Generation Control $(\mathrm{AGC})$, and reserve markets. By applying the proposed method, not only trading loss over the whole scheduling horizon can be controlled, but also the amount of imposed loss during every time period can be reduced. An illustrative case study is provided for further analysis.
\end{abstract}

(C) 2018 Sharif University of Technology. All rights reserved.

\section{Introduction}

In financial literature, risk management techniques include at least two aspects: risk assessment and risk control. Adopting an appropriate risk measure, which best reflects the risk with respect to decision maker's attitude, is categorized within risk assessment. In this area, variance criterion and expected utility have been attractive measures used for several decades. Portfolio optimization has come a long way since its appearance. Developments in this area are stimulated by two basic requirements:

\footnotetext{
*. Corresponding author. Fax: +9823 33654089 E-mail addresses: s.bazmohammadi@semnan.ac.ir (S. Bazmohammadi); aakbari@semnan.ac.ir (A. Akbari Foroud); najme_bazmohammadi@yahoo.com (N. Bazmohammadi)
}

1. Adequate modeling of utility functions, risks, and constraints;

2. Efficiency, i.e., ability to handle a large number of instruments and scenarios [1].

Under this inspiration, downside risk measures such as Value at Risk (VaR) and Conditional Value at Risk (CVaR) are introduced [1]. Downside risk measures focus on undesirable aspects of risk and evaluate the profits under a specified level or target. On the contrary, variance measure incorporates information from both tails of profit distribution, so it is affected by high gains as well as high losses. Although introducing VaR filled a large gap to assess the extreme risk associated with a trading plan, it was later demonstrated that VaR exhibited some undesirable mathematical characteristics. It was demonstrated by Artzner that risk measures had to include some properties to reflect the commonly accepted rational behavior of a decision maker [2]. What VaR lacks is sub-additivity and 
convexity, misleading the investors by providing no motivation for diversifying their investments. Fortunately, CVaR measure is consistent with diversification strategies in portfolio optimization. In addition, CVaR can be combined with analytical or simulation-based methods and in cases that the uncertainty is modeled by a finite number of scenarios, the problem can be reduced to linear programming [1]. Risk control can be accomplished by means of two methods: "hedging," which is a technique to offset the particular source of risk, and "diversification" [3]. In an electricity market, forward contracts, which omit the risk of price volatility, are available for hedging against risk, whereas a variety of spot markets such as Automatic Generation Control (AGC) and reserve markets are provided for making diversification in the trading plan. A combination of different types of transactions for trading energy in several markets is called energy portfolio and the problem of constructing an optimal trading plan that specifies the contribution of GENCO to each market is called portfolio optimization problem. Various aspects of risk management have been applied to the electricity market. In [4-13] it is aimed to manage risk from a supplier or a consumer (distribution company) point of view. The risk management technique proposed in [46] is based on variance measure [7] proposes a bi-level multi-objective bidding strategy through a combination of maximizing profit and minimizing risk with the help of a risk-tolerance parameter, which reflects the GENCO's preference toward the risk. CVaR measure is applied to the strategies proposed in [8-13]. In all of the previous works, GENCO's financial risk is evaluated over the total planning horizon, called long-term in this paper, and the risk aversion preferences relating to each period are ignored. However, it was stated by Artzner in 2007 [14] that modeling the investment risk, evolving over several periods of uncertainty, was different from one-period risk and the actual time evolution of risk should be taken into account through a relevant riskadjusted measurement, so that more than the distribution of final profit of a strategy was provided for risk management. The main reason of Artzner was not only considering the actual consequences of "bad events" at the end of the same periods, which were ignored in longterm models of risk management, but also the necessity of taking into account the information availability in some decision making periods that might require intermediate tactical actions to handle sequences of unknown future.

In a restructured electricity industry, a generation company (GENCO) has to participate in various energy and ancillary services auctions and compete with other producers in order to sell its products. The extremely volatile prices of this competitive market are not only depend on the market players' strategies, but also affected by special characteristics of electricity as a commodity, which cannot be easily stored and transported. Furthermore, stochastic nature of the demand and its dependency on weather conditions, probable failures of generating units, outages of transmission lines, etc., extremely influence the market prices. On the other hand, GENCO's trading plans have to be made before clearing successive hourly market sessions by independent system operator and observing the realization of market prices. The contributions of the proposed method for optimal portfolio construction are as follows:

1. Introducing a multi-period risk management technique. In addition to managing long-term risk, the proposed strategy provides suppliers with an extra measuring and controlling tool to reduce financial risk during shorter intervals, called short-term risk management in this paper. To the best of the authors' knowledge, this study has never been done before;

2. Utilizing fuzzy satisfaction theory to model risk preferences. In order to attain appropriate modelling of short-term risk preferences of the decision maker, fuzzy satisfaction theory is utilized to incorporate the vague and fuzzy nature of human judgment. Therefore, the risk preferences of the GENCOs are well modelled and incorporated into the stochastic decision making problem;

3. Managing market risk by hedging and diversification tools. The proposed portfolio optimization approach has the capability of reducing risks by making "diversification" in the trading plan and "hedging" through forward contracts. This method allocates optimal trading proportion to multiple spot markets while the possibility of signing forward contracts and involvement in arbitrage opportunities is also considered;

4. Precise modeling of the production units and utilizing advanced CPLEX solver. By considering prevailing operational constraints of the thermal units and utilizing the advanced CPLEX solver under GAMS for solving this stochastic mixed-integer programming problem, the developed algorithm is made suitable for practical situations.

The rest of the paper is organized as follows. Decision making framework and price characterization are presented in Section 2. The objectives of optimal unit commitment and self-scheduling of a producer are formulated in Section 3. Section 4 provides a detailed case study for further illustration of the proposed method. Finally, concluding remarks are given in Section 5. 


\section{Decision framework}

In this paper, the considered uncertainty sources include prices for the day-ahead energy market, automatic generation control, and reserve markets. Uncertainty in market clearing prices is handled by treating prices as stochastic variables that are modeled by their probability density functions. It is assumed that market clearing price and its variance are inputs of the proposed model. Furthermore, as it is generally preferred in stochastic programming, instead of dealing with a continuous probability distribution function for modeling the price of each time period, continuous variable is substituted with a set of discrete outcomes, which are called scenarios, as it is shown in Figure 1. The abstract structure of scenarios can be represented by a scenario tree.

\subsection{Scenario generation}

In this paper, we use Monte Carlo simulation that utilizes statistical sampling techniques for generating adequate number of scenarios with the same probability. Therefore, a probabilistic approximation of the solution to the mathematical model can be properly produced. Large number of scenarios may result in intractability of the optimization problem. Consequently, scenario reduction techniques are generally employed to trim down the number of scenarios while maintaining, as much as possible, the stochastic properties of the original scenario tree [15]. In dealing with stochastic programming, which has the reputation for being computationally difficult to solve, a natural temptation is to substitute a much simpler problem that replaces all of the random variables by their expected values. In this situation, the Value of Stochastic Solution (VSS) is a concept that precisely measures how good the stochastic solution is. Consider the problem of maximizing profit when stochastic market prices are replaced by their expected values, called $E V$, as shown

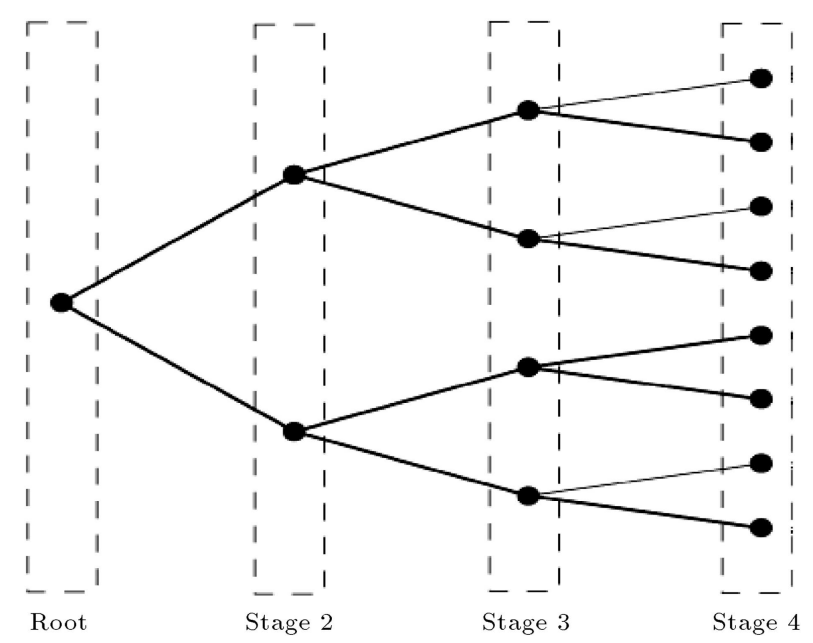

Figure 1. Multi-stage price scenario tree. by:

$$
E V=\text { Maximize } f\left(x, E\left(\lambda_{t}\right)\right) .
$$

In this equation, $f$ denotes the profit function, $x$ is the decision vector, which belongs to the feasible region, and $E\left(\lambda_{t}\right)$ shows the expected value of hourly prices. Let $x^{*}$ be the optimal solution to Eq. (1) and EEV the expected result of using $x^{*}$ according to:

$$
E E V=E_{\lambda}\left[f\left(x^{*}, \lambda_{t}\right)\right] \text {. }
$$

Then, $V S S$ can be calculated as the difference between expected profit of stochastic model and $E E V$ [16]. If the value of $V S S$ is high, dealing with uncertainty really matters.

\section{Problem formulation}

Objective function of the optimization problem is formulated in a stochastic mixed integer programming framework as follows. Eq. (3) consists of two parts: profit maximization and risk control; the latter term is incorporated by factor $\alpha$, representing the decision maker's risk preferences. A risk-lover GENCO selects minimum value $(\alpha=0)$ while a conservative producer prefers higher amounts:

$$
\begin{aligned}
\operatorname{Max} E_{\omega} & \left\{\sum_{i=1}^{N_{I}} \sum_{t=1}^{N_{T}} \Theta_{t \omega i}\left(R E_{t \omega}(i), R S_{t \omega}(i)\right)\right\} \\
& +\sum_{i=1}^{N_{I}} \phi(i)-E_{\omega}\left\{\sum_{i=1}^{N_{I}} \sum_{t=1}^{N_{T}} C_{t \omega}(i)\right\} \\
& +\alpha . \text { Risk Measure. }
\end{aligned}
$$

\subsection{Profit maximization}

Generally, the revenue of a GENCO can be divided into two terms:

1. Stochastic revenue obtained from selling energy and ancillary services in the pool market;

2. Deterministic revenue coming from trading energy under contracts.

In Eq. (3), $\Theta_{t \omega i}$ is used to calculate the stochastic part and $\Phi(i)$ denotes revenue of forward contracts. Production consists of fixed cost, variable cost, time varying start-up cost, and shutdown cost. $R E_{t \omega}(i)$ represents revenues of energy market that are calculated through multiplying the amount of power allocated to the energy market at time $t$ and scenario $\omega\left(P_{t \omega}^{E}\right)$ by the price of energy market $\lambda_{t \omega}^{E}(i)(\$ / \mathrm{MWh}) . R S_{t \omega}(i)$ denotes the revenues obtained through ancillary services markets, including automatic generation control, spinning reserve, and non-spinning reserve. Finally, $\Theta_{t \omega i}$ that is the stochastic part of the GENCO's revenue can be calculated by summation of $R E_{t \omega}(i)$ and $R S_{t \omega}(i)$. 


\subsubsection{Ancillary services market}

The formulation that is presented in this paper is based on simultaneous clearing of energy and ancillary services markets, which includes Automatic Generation Control (AGC), spinning reserve market, and non-spinning reserve market. In case these markets clear sequentially, the proposed method can be used to schedule generation units for energy market and, then, execute them again by applying energy results. Consequently, realization of day-ahead market clearing prices improves scheduling of the units for ancillary services market. Although reference [5] develops a probabilistic based approach to model whether reserves are called and generated, in this paper, it is assumed that market has enough liquidity to allow generation units to sell optimal amounts they desire. It is assumed that ancillary services are the products that generation companies bid in electricity market in steady state and fluctuations are not in the scope of this work. For more information, Lei et al. [17-19] address the problem of devising an optimal AGC for interconnected networks based on a stochastic multi-agent strategy. It should be noted that although the amount of power sold in ancillary services market is not comparable with the amount of power traded in energy market, ancillary services are usually generated at low cost [20]. Eqs. (4)(10) model the maximum power that can be devoted to ancillary services market. In these equations, $R_{t \omega}^{x}(i)$, $x=\{A, S, N U, N D\}$ stands for the amount of power allocated to AGC, spinning reserve, non-spinning reserve when unit $i$ is on, and non-spinning reserve when it is off. Also, $U_{t}(i)$ is unit status indicator, which equals 1 if unit $i$ is on at time $t$ and 0 otherwise. According to Eq. (4), $R_{t \omega}^{A}(i)$ is limited by maximum capability of unit $i$ for participating in AGC market and regulating limits. In this equation, $A G C_{t}(i)$ is a binary variable that indicates involvement in the AGC market:

$$
\begin{array}{ll}
R_{t \omega}^{A}(i) \leq \min \left\{R_{\max }^{A}(i) A G C_{t}(i), A L\right\}, & \forall i, t, \omega, \\
R_{t \omega}^{S}(i) \leq R_{\max }^{S}(i) U_{t}(i), & \forall i, t, \omega, \\
R_{t \omega}^{N U}(i) \leq R_{\max }^{N}(i) U_{t}(i), & \forall i, t, \omega, \\
R_{t \omega}^{N D}(i) \leq R_{\max }^{N}(i)\left(1-U_{t}(i)\right), & \forall i, t, \omega, \\
R_{t \omega}^{N U}(i)+R_{t \omega}^{N D}(i) \leq R_{\max }^{N}(i), & \forall i, t, \omega .
\end{array}
$$

It is worth noticing that for selling power to AGC and spinning reserve markets, units have to be synchronous with the system, while non-spinning reserve can be provided by both off-line and on-line generating resources that can increase their output power within a certain time.

$$
\begin{array}{ll}
A G C_{t}(i) \leq U_{t}(i), & \forall i, t, \\
A G C_{t}(i) \in\{0,1\}, & \forall i, t .
\end{array}
$$

Total power that can be allocated to various ancillary services markets is constrained by ramping limits; Eq. (12) enforces this limitation on the summation of power allocated to different ancillary services markets as stated in Eq. (11). $P_{t \omega}^{q}$ is the output power of unit $i$, excluding power to be delivered to ancillary services. Also, $R U(i)$ and $S U(i)$ in Eq. (12) represent rampup limit and start-up ramp limit of unit $i$, respectively. Eq. (13) constrains the involvement in energy market and ancillary services markets to maximum output power of each generating unit. Finally, revenue obtained from trading energy in ancillary services markets, $R S$, can be calculated by Eq. (14).

$$
\begin{aligned}
& R_{t \omega}^{A S}(i)=R_{t \omega}^{A}(i)+R_{t \omega}^{S}(i)+R_{t \omega}^{N U}(i)+R_{t \omega}^{N D}(i), \\
& \forall i, t, \omega, \\
& R_{(t+1) \omega}^{A S}(i)+P_{(t+1) \omega}^{q}(i)-P_{t \omega}^{q}(i) \leq\left[R U(i) U_{t}(i)\right. \\
& \left.+S U(i) w_{(t+1)}(i)\right], \quad \forall i, t, \omega \\
& R_{t \omega}^{A S}(i)+P_{t \omega}^{q}(i) \leq P_{\max }(i), \quad \forall i, t, \omega, \\
& R S_{t \omega}(i)=\sum_{e=A, S} \lambda_{t \omega}^{e}(i) R_{t \omega}^{e}(i)+\lambda_{t \omega}^{N}(i)\left(R_{t \omega}^{N U}(i)\right. \\
& \left.+R_{t \omega}^{N D}(i)\right), \quad \forall i, t, \omega .
\end{aligned}
$$

\subsubsection{Contract market}

A producer may decide to buy electric energy through forward contracts to sell in the pool market with the hope of increasing its profit, but at a cost of higher risk, or sell the produced energy in contracts to avoid the inherent risk of price volatility of the pool market. Forward contracts consist of blocks of power spanning over a period of time with a specified price in $\$ / \mathrm{MWh}$. Modeling of price behavior in some markets, such as European Energy Exchange [21] and Iberian Electricity Market Operator [22], has shown that as the quantity sold/bought in contracts increases, the specified price may decrease/increase. This stepwise price form reflects both high volatility and limited liquidity of future markets [11]. In this paper, we assume that the GENCO can sell/buy energy to/from forward contracts. It is also possible to buy energy from contract market and sell in the pool. For the sake of simplicity, it is assumed that selling and buying cannot be done in the same contract. Following equations are used for modeling necessary constraints associated with utilizing forward contracts: 


$$
\begin{aligned}
& P_{t \omega}^{E}(i)=P_{t \omega}^{q}(i)+\sum_{c=1}^{N C^{B}} \sum_{b=1}^{N b^{B}} P_{c b}^{B}(i)-\sum_{c=1}^{N C^{S}} \sum_{b=1}^{N b^{S}} P_{c b}^{S}(i), \\
& -P_{t \omega}^{D}(i) \\
& \forall i, t, \omega \\
& \sum_{b=1}^{N b^{S}} P_{c b}^{S}(i) \leq U_{c S}(i) \sum_{b=1}^{N b^{S}} P_{c b}^{S, \max }(i), \quad \forall c, i \\
& \sum_{b=1}^{N b^{B}} P_{c b}^{B}(i) \leq U_{c B}(i) \sum_{b=1}^{N b^{B}} P_{c b}^{B, \max }(i), \quad \forall c, i \\
& P_{c b}^{B} \leq P_{c b}^{B, \max }, \quad \forall c, b, \\
& P_{c b}^{S} \leq P_{c b}^{S, \max }, \quad \forall c, b, \\
& U_{c B}(i)+U_{c S}(i) \leq 1, \quad \forall c, i, \\
& P_{t \omega}^{g}(i) \in \Pi, \quad \forall t, \omega, i, \\
& P_{c b}^{B}(i), P_{c b}^{S}(i) \geq 0, \quad \forall c, b, \\
& P_{t \omega}^{E}(i) \geq 0, \quad \forall t, \omega, i, \\
& U_{c B}(i), U_{c S}(i) \in\{0,1\}, \quad \forall c, i .
\end{aligned}
$$

The amount of power sold in the energy market, $P_{t \omega}^{E}$, is denoted by Eq. (15) and is expressed as the summation of output power of unit $i$, total power traded through forward contracts, and what is allocated to its own loads (e.g., GENCO's demand or other obligations) and ancillary services market. $P_{c b}^{S} / P_{c b}^{B}$ represents the power sold/purchased through block $b$ of forward contract c. Also, $U_{c S} / U_{c B}$ is an indicator of selling/buying through bilateral contract $c$. Eqs. (16)-(19) specify the maximum power which can be traded in contracts, so that the power allocated to each block does not exceed the maximum capacity of that block in contract $c\left(P_{c b}^{B, \max }, P_{c b}^{S, \max }\right)$. Furthermore, the summation of power allocated to blocks of contract $c$ should be constrained by the maximum power that can be sold and purchased through bilateral contract. Constraint (20) states that power cannot be sold and bought with the same forward contract. Moreover, Eqs. (21)-(24) are used for variable declaration. Symbol $\Pi$ in Eq. (21) stands for generated power constraints discussed in the next section. Finally, revenue obtained from trading energy through contracts can be formulated as follows:

$$
\begin{aligned}
\phi(i)= & \sum_{c=1}^{N C} \sum_{b=1}^{N b^{S}} \lambda_{c b}^{S}(i) P_{c b}^{S}(i) L_{c}^{S} \\
& -\sum_{c=1}^{N C} \sum_{b=1}^{N b^{B}} \lambda_{c b}^{B}(i) P_{c b}^{B}(i) L_{c}^{B}
\end{aligned}
$$

$\lambda_{c b}^{S}(i)$ and $\lambda_{c b}^{B}(i)$, respectively, denote prices of block $b$ for selling and buying in contract $c$ where $L_{c}$ is time duration of each forward contract. It should be noticed that the revenue of contract markets is independent of price scenarios, which makes them suitable choices to hedge against pool price uncertainty.

\subsubsection{Production cost}

In this paper, production cost consists of fixed cost plus variable cost in piecewise linearized production cost curve plus time varying start-up cost and shutdown cost, as illustrated in Eq. (26). Generating power of unit, $i$, is presented by Eq. (27):

$$
\begin{aligned}
C_{t \omega}(i)= & A(i) U_{t}(i)+\sum_{m=1}^{N_{m}} p_{t \omega m}(i) S L_{m}(i) \\
& +w_{t}(i) S T C_{t}(i)+z_{t}(i) S C(i), \quad \forall i, t, \omega, \\
P_{t \omega}^{g}(i)= & p_{\min }(i) U_{t}(i)+\sum_{m=1}^{N_{m}} p_{t \omega m}(i), \quad \forall i, t, \omega .
\end{aligned}
$$

In these equations, the slope of power block, $m$, for unit, $i$, in piecewise linearized production cost is defined by $S L_{m}(i)$ and the number of blocks is represented by $N_{m}$. $A(i), S T C_{t}(i)$, and $S C(i)$ represent the fixed cost, time varying start-up cost, and shutdown cost of the $i$ th unit. $z_{t}(i) / w_{t}(i)$ is a binary variable that equals 1 if the unit is shut down/started up at time $t$ and 0 otherwise. Furthermore, $P_{t \omega m}(i)$ denotes the power generated in each block and $P_{t \omega}^{g}(i)$ is the output power of unit, $i$, which is the sum of the power generated in each block plus minimum power output $P_{\min }(i)$.

Operating thermal unit is constrained by some limitations. The set of Formulations (28) and (29) is utilized to model ramp rate limits:

$$
\begin{aligned}
& P_{(t+1) \omega}^{g}(i)-P_{t \omega}^{g}(i) \leq\left[R U(i) U_{t}(i)+S U(i) w_{t+1}(i)\right] \\
& \quad \forall i, t, \omega \\
& P_{t \omega}^{g}(i)-P_{(t+1) \omega}^{g}(i) \leq\left[R D(i) U_{t+1}(i)+S D(i) z_{t+1}(i)\right] \\
& \quad \forall i, t . \omega .
\end{aligned}
$$

Minimum uptime has been modeled through Eqs. (30)(32). Eqs. (30) and (31) are utilized in order to maintain unit $i$ on with respect to its minimum uptime $M U(i)$ and the number of hours it has been on at the beginning of the scheduling period, $T U_{0}(i)$. Minimum uptime limitation for time periods other than the first period is satisfied by (32):

$$
\sum_{t=1}^{U T(i)}\left(1-U_{t}(i)\right)=0, \quad \forall i
$$




$$
\begin{aligned}
& U T(i)=\max \left[0, \min \left[N_{T},\left[M U(i)-T U_{0}(i)\right] U_{t 0}(i)\right]\right] \\
& \forall i, \\
& w_{t}(i)+\sum_{m=t+1}^{\min \left[N_{T}, t+M U(i)-1\right]} z_{m}(i) \leq 1 \\
& \forall i, t=U T(i)+1, \cdots, T .
\end{aligned}
$$

Minimum downtime has been modeled through Eqs. (33)-(35). Eqs. (33) and (34) are utilized in order to maintain unit $i$ off with respect to its minimum downtime, $M D(i)$, and the number of hours it has been on at the beginning of the scheduling period, $T C_{0}(i)$. Minimum downtime limitation for time periods other than the first period is satisfied by (35):

$$
\begin{aligned}
& \sum_{t=1}^{D T(i)}\left(U_{t}(i)\right)=0, \quad \forall i, \\
& D T(i)=\max \left[0, \min \left[N_{T},\left[M D(i)-T C_{0}(i)\right]\right.\right. \\
& \left.\left.\left(1-U_{t 0}(i)\right)\right]\right], \quad \forall i, \\
& z_{t}(i)+\sum_{m=t+1}^{\min \left[N_{T}, t+M D(i)-1\right]} w_{m}(i) \leq 1, \\
& \forall i, t=D T(i)+1, \cdots, T .
\end{aligned}
$$

Maximum/minimum output power constraints are modeled by Eqs. (36) and (37):

$$
\begin{aligned}
& P_{t \omega}^{g}(i) \leq p_{\max }(i) U_{t}(i), \quad \forall i, t, \omega, \\
& p_{\min }(i) U_{t}(i) \leq P_{t \omega}^{g}(i), \quad \forall i, t, \omega .
\end{aligned}
$$

Furthermore, Eqs. (38)-(45) make linear expression of exponential start-up cost. In order to define start-up cost as a function of hours that the unit has been off, $T C_{t}(i)$ is utilized as an off-time counter. The value of $M$ in Eq. (38) should be as small as possible, but large enough for a good mixed integer programming formulation; in this case, $M=N_{T}+T C_{0}$ [23]. $v_{t k}(i)$ is a binary variable that equals 1 if unit $i$ is started up in segment $k$ of the start-up curve. $N_{k}$ represents the number of intervals of start-up cost curve. Moreover, $q_{t}(i)$ is a dummy variable and is used when either unit $i$ is off at hour $t$ or it is started up at hour $t$ and has been off for $N_{k}$ hours [24]. Furthermore, $S T C_{t}$ denotes time-varying start-up cost, in which $S T_{k}(i)$ represents start-up cost at segment $k$ in the stair-wise start-up curve:

$$
\begin{array}{ll}
0 \leq T C_{t}(i) \leq M\left[1-U_{t}(i)\right], & \forall i, t \\
T C_{t-1}(i)-T C_{t}(i) \leq M U_{t}(i)-1, & \forall i, t, \\
T C_{t}(i)-T C_{t-1}(i) \leq 1, & \forall i, t
\end{array}
$$

$$
\begin{array}{lc}
\sum_{k=1}^{N_{k}} v_{t k}(i)=w_{t}(i), & \forall i, t, \\
T C_{t-1}(i)=\sum_{k=1}^{N_{k}-1} k v_{t k}(i)+q_{t}(i), & \forall i, t, \\
q_{t}(i) \leq M\left[v_{t N_{k}}(i)-U_{t}(i)+1\right], & \forall i, t \\
q_{t}(i) \geq N_{k} v_{t N_{k}}(i), & \forall i, t, \\
S T C_{t}(i)=\sum_{k=1}^{N_{k}} S T_{k}(i) v_{t k}(i), & \forall i, t .
\end{array}
$$

Finally, Eqs. (46)-(48) are used for variables declaration [23-25]:

$$
\begin{array}{lr}
U_{t+1}(i)-U_{t}(i)=w_{t+1}(i)-z_{t+1}(i), & \forall i, t, \\
w_{t}(i)-z_{t}(i) \leq 1, & \forall i, t, \\
U_{t}(i), w_{t}(i), z_{t}(i), v_{t k}(i) . &
\end{array}
$$

\subsection{Multi-period risk management based on conditional value at risk}

Conditional Value at Risk (CVaR) can be defined as the conditional expectation of profits lower than a threshold called VaR. It should be noted that the profit might be negative and, thus, in effect, constitute a loss. At the confidence level $\beta, \mathrm{CVaR}$ is the expected value of portfolio profits in the $(1-\beta) \times 100 \%$ worst cases. Three common values of $\beta$ are $0.90,0.95$, and $0.99[2]$.

In this paper, portfolio optimization of a GENCO is handled by assessment of market price risk using the multi-period CVaR measure. Since stochastic nature of volatile market prices is handled by considering enough number of price scenarios, for each realization of market prices, a value of profit for the GENCO is obtained. Consequently, a profit distribution is generated by considering total planning horizon and 24 profit distributions are produced for modelling of the profit of each time period. Long-term risk management deals with the CVaR of the profit distribution function of the total planning horizon (TCVaR). On the other hand, the HCVaRs are calculated based on hourly profit distributions and have been used to control shortterm risk. Risk of profit variability is modeled through CVaR methodology by Eqs. (49)-(56). Eqs. (49)-(52) model the short-term risk preferences of the decision maker while Eqs. (53)-(56) are used to control longterm risk. Short-term risk measure is defined in Eq. (49):

$$
\text { Risk measure }=\sum_{i=1}^{N_{I}} \sum_{t=1}^{N_{T}} \rho_{t}(i) H C V a R_{t}(i),
$$




$$
\begin{aligned}
& \operatorname{HCVaR}_{t}(i)=\left\{\eta_{t}^{s h}(i)-\left(\frac{1}{1-\beta^{s h}}\right) \sum_{\omega=1}^{N \omega} \pi_{\omega} \mu_{t \omega}^{s h}(i)\right\} \\
& \forall i, t, \\
& \Theta_{t \omega i}\left(R E_{t \omega}(i), R S_{t \omega}(i)\right)+\phi(i)-C_{t \omega}(i) \\
& -\eta_{t}^{s h}(i)+\mu_{t \omega}^{s h}(i) \geq 0, \quad \forall t, \omega, i, \\
& \mu_{t \omega}^{s h}(i) \geq 0, \quad \forall t, \omega, i, \\
& T C V a R=\eta^{L}-\left(\frac{1}{1-\beta^{L}}\right) \sum_{\omega=1}^{N \omega} \pi_{\omega} \mu_{\omega}^{L}, \\
& T C V a R \geq \Omega_{L 0}, \\
& \sum_{i=1}^{N_{I}}\left\{\sum_{t=1}^{N_{T}}\left\{\Theta_{t \omega i}\left(R E_{t \omega}(i), R S_{t \omega}(i)\right\}+\phi(i)-C_{t \omega}(i)\right\}\right. \\
& -\eta^{L}+\mu_{\omega}^{L} \geq 0, \quad \forall \omega, \\
& \mu_{\omega}^{L} \geq 0, \quad \forall \omega .
\end{aligned}
$$

$H C \operatorname{VaR}_{t}(i)$ is the short-term CVaR of profit of unit $i$ in the time period $t$ and TCVaR is long-term CVaR of the GENCO's profit. It should be noted that longterm risk control is achieved by imposing a limitation $\Omega_{L 0}$ on the long-term CVaR while the short-term risk preferences of the GENCO for unit $i$ are incorporated into the model by adopting risk aversion coefficients $\rho_{t}(i)$. Since each Locational Marginal Price (LMP) could be affected by various local means such as actions of competitors and demand behavior of the specific area, the corresponding risk management technique may be different from one unit to another, so risk management strategies have been devised in accordance with risk preferences of each unit. Reference [26] tries to evaluate the relation between profit and risk aversion based on Internal Revenue Service (IRS) data and estimates changes in risk aversion. It states that risk aversion has a decreasing trend as the wealth increases. Consequently, risk aversion of a GENCO in every market session can be assumed dependent on its corresponding price. In other words, the higher the price, the lower the risk aversion would be. From another point of view, volatility in price behavior can be used to determine decision making strategy. Typically, price spikes appear in extreme situations such as bad weather or unexpected outages in the network. In these situations, by devising a bad bidding strategy, a loss of millions of dollars within a few days or even hours is unavoidable. To reduce bidding risks, a GENCO may prefer to sell power when the market uncertainties are

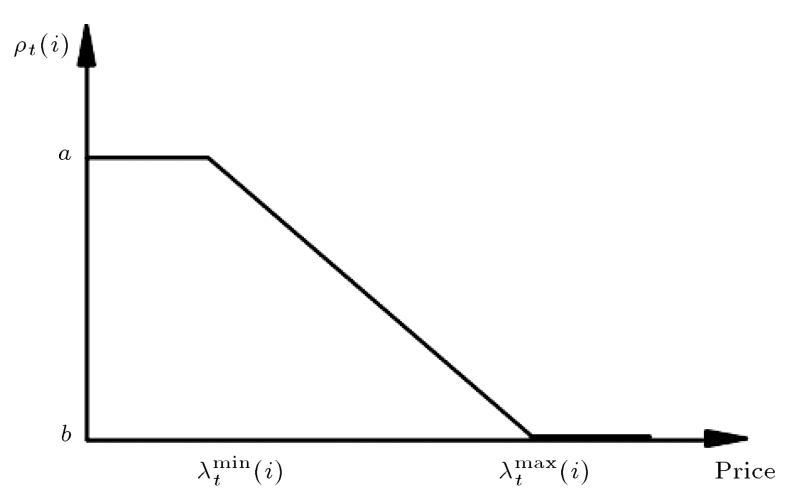

Figure 2. Fuzzy membership function.

high to capture potential price spikes and buy power when the market uncertainties are low to avoid risks [4]. In this paper, fuzzy satisfaction theorem based on linear membership function is utilized to model risk preferences of the decision maker. As depicted in Figure 2, fuzzy membership function produces a risk factor between $a$ and $b$ proportional to expected price of each hour; $a$ represents maximum risk aversion and $b$ corresponds to risk loving attitude. $\lambda_{t}^{\max } / \lambda_{t}^{\min }$ is the maximum/minimum specified price greater/lower than the price to which the minimum/maximum risk aversion coefficient is assigned.

In the following section, the effectiveness of the proposed method is examined by comparing the results of applying risk neutral, long-term risk management, and the proposed strategy in the optimal portfolio selection problem. Figure 3 represents the flowchart of the execution steps of the applied methodology.

\section{Numerical results and discussion}

The model presented in the previous sections is illustrated through a case study. The considered GENCO owns three thermal units with total maximum generation capacity of $950 \mathrm{MW}$. Technical specifications of the units such as output power, rating capability, minimum uptime/downtime, operation cost, start-up and shutdown costs, etc. are provided in Tables 13. In this paper, the considered ancillary services market includes Automatic Generation Control (AGC), spinning reserve, and non-spinning reserve. Energy and ancillary services are traded in a pool-based electricity market where producers are awarded by the market clearing prices corresponding to each market. Four types of forward contracts are provided for each generation unit; selling and buying contracts in peak and off-peak hours. Since forward contracts are usually traded over longer periods of time, peak contracts cover 8 hours and off-peak contracts are defined over a span of 16 hours. It is assumed that each contract can consist in up to two $30 \mathrm{MW}$ blocks with the price behavior described in Section 3.1.2. Available contracts and 


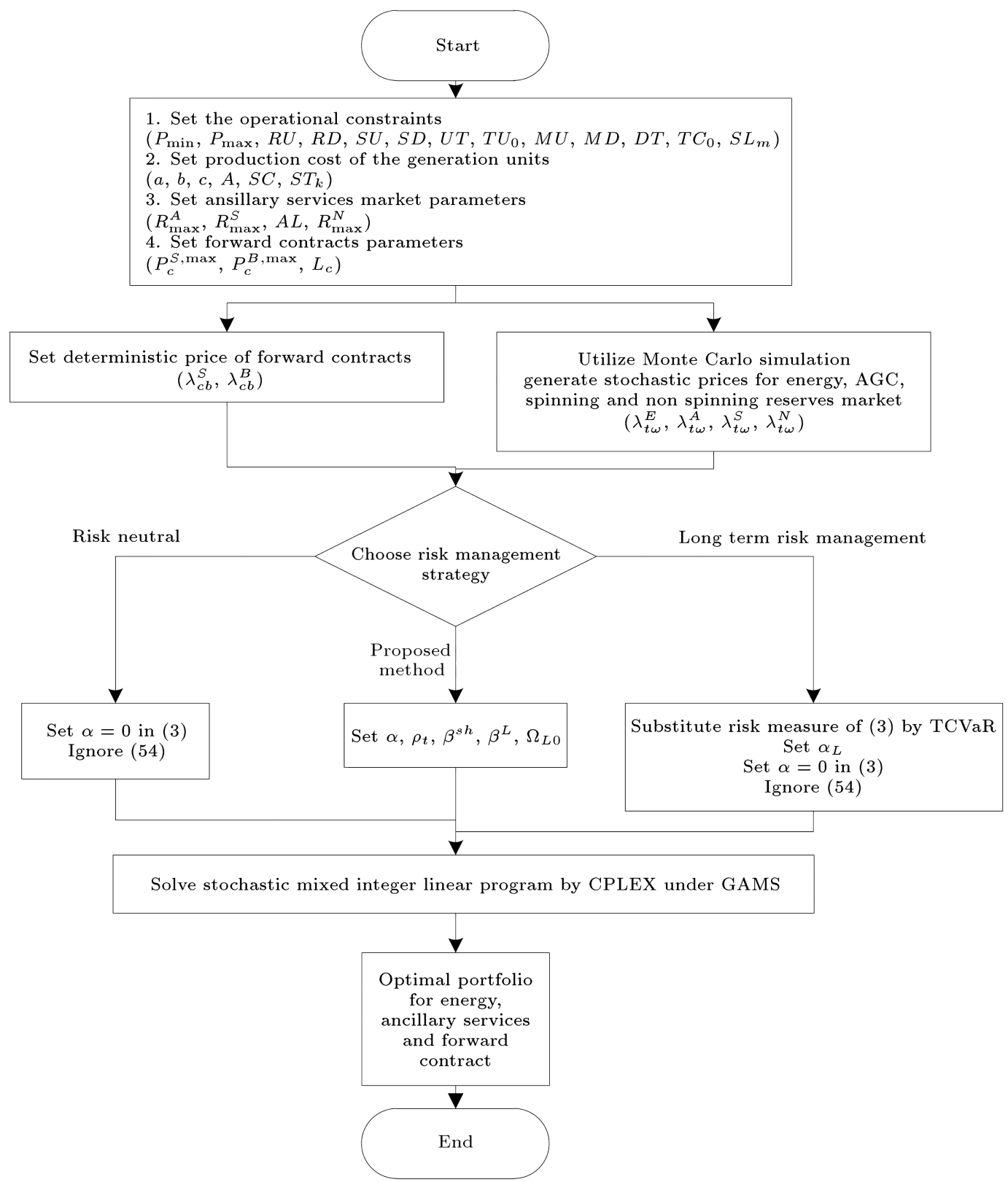

Figure 3. Flowchart of the execution steps of the applied methodology.

Table 1. Cost data of the units.

\begin{tabular}{ccccccc}
\hline $\begin{array}{c}\text { Unit } \\
\text { no. }\end{array}$ & $\begin{array}{c}\boldsymbol{P}_{\max } \\
(\mathbf{M W})\end{array}$ & $\begin{array}{c}\boldsymbol{P}_{\min } \\
(\mathbf{M W})\end{array}$ & $\begin{array}{c}\boldsymbol{a} \\
(\$ / \mathbf{M w h})\end{array}$ & $\begin{array}{c}\boldsymbol{b} \\
(\$ / \mathbf{M w h})\end{array}$ & $\begin{array}{c}\boldsymbol{c} \\
(\$)\end{array}$ & $\begin{array}{c}\boldsymbol{S C} \\
(\$)\end{array}$ \\
\hline 1005 & 300 & 100 & 0.10875 & 24.8875 & 6.78 & 115 \\
1010 & 300 & 100 & 0.010875 & 25.8875 & 6.78 & 96.4 \\
1011 & 350 & 100 & 0.003 & 26.76 & 32.96 & 126.3 \\
\hline
\end{tabular}

Table 2. Stair-wise start-up cost $(\$)$

\begin{tabular}{cccccc}
\hline Unit no. & $\boldsymbol{S T}_{\mathbf{1}}$ & $\boldsymbol{S T}_{\mathbf{2}}$ & $\boldsymbol{S T}_{\mathbf{3}}$ & $\boldsymbol{S T}_{\mathbf{4}}$ & $\boldsymbol{S T}_{\mathbf{5}}$ \\
\hline 1005 & 100.88 & 200.76 & 300.32 & 310.45 & 315.87 \\
1010 & 102.23 & 205.43 & 303.88 & 309.34 & 316.55 \\
1011 & 107.57 & 210.56 & 308.34 & 312.49 & 317.62 \\
\hline
\end{tabular}


Table 3. Technical characteristics of the units.

\begin{tabular}{ccccccc}
\hline $\begin{array}{c}\text { Unit } \\
\text { no. }\end{array}$ & $\begin{array}{c}\boldsymbol{R U} \\
(\mathbf{M W} / \mathbf{h})\end{array}$ & $\begin{array}{c}\boldsymbol{R} \boldsymbol{D} \\
(\mathbf{M W} / \mathbf{h})\end{array}$ & $\begin{array}{c}\boldsymbol{S U} \\
(\mathbf{M W} / \mathbf{h})\end{array}$ & $\begin{array}{c}\boldsymbol{S D} \\
(\mathbf{M W} / \mathbf{h})\end{array}$ & $\begin{array}{c}\boldsymbol{D T} \\
(\mathbf{h})\end{array}$ & $\begin{array}{c}\boldsymbol{U} \boldsymbol{T} \\
(\mathbf{h})\end{array}$ \\
\hline 1005 & 50 & 50 & 100 & 100 & 3 & 4 \\
1010 & 50 & 50 & 100 & 100 & 3 & 4 \\
1011 & 75 & 75 & 150 & 150 & 3 & 4 \\
\hline
\end{tabular}

Table 4. Forward contracts data.

\begin{tabular}{ccccc}
\hline \multicolumn{2}{c}{$\begin{array}{c}\text { Contract } \\
\text { type }\end{array}$} & $\boldsymbol{P}_{\boldsymbol{c}}$ & \multicolumn{2}{c}{$\boldsymbol{\lambda}_{\boldsymbol{c}}(\$ \mathbf{M W h})$} \\
\cline { 3 - 4 } Buy & Off-peak & 60 & Block 1 & Block 2 \\
\cline { 3 - 4 } & Peak & 60 & 21.53 & 21.63 \\
\multirow{4}{*}{ Sell } & & & \\
& & & & \\
& Off-peak & 60 & 22.661 & 22.761 \\
& Peak & 60 & 28.461 & 28.361 \\
\hline
\end{tabular}

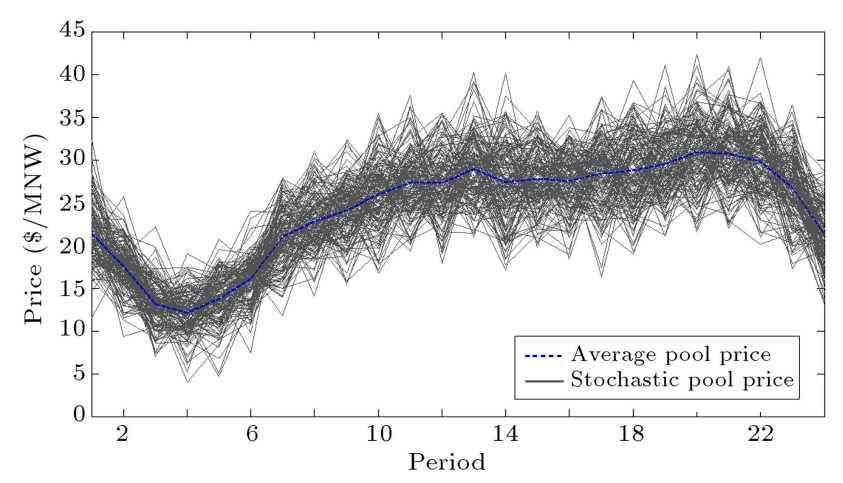

Figure 4. Stochastic energy price.

their associated prices are given in Table 4. Stochastic prices for energy and ancillary services markets are provided by the Monte Carlo simulation method. The sufficient number of price scenarios is determined to be 160 scenarios to ensure traceability and maintain stochastic nature of the problem. Energy market price is presented in Figure 4. Prices for other markets are produced similarly.

The assumed short-term risk aversion coefficients based on fuzzy membership function are provided in Table 5. The parameters ' $a$ ' and ' $b$ ' are considered
1 and 0 , respectively, where $\lambda_{t}^{\max }$ and $\lambda_{t}^{\min }$ are set proportional to the expected price. Furthermore, an appropriate $\Omega_{L 0}$ is picked up so that the amount of long-term CVaR is constrained to the minimum accepted value. The effectiveness of the proposed method is illustrated through analyzing the results of various portfolio selection methods considering risk neutral strategy, long-term risk management technique, and the proposed method. Each of these three strategies is examined on four cases as represented below:

- Case A: Unit commitment and scheduling for energy market;

- Case B: Optimal portfolio selection for involvement in energy market and signing forward contracts;

- Case C: Optimal portfolio selection for involvement in energy and ancillary services markets;

- Case D: Optimal portfolio selection for involvement in energy and ancillary services markets and forward contracts.

Since Case D is the most comprehensive case, Value of Stochastic Solution $(V S S)$ is calculated in this case. Expected profit of risk neutral strategy based on stochastic method is $\$ 31617$, which is by $\$ 4078$ greater than the expected profit obtained solving the $E E V$ problem. Consequently, VSS is $12.9 \%$, which emphasizes the importance of modelling uncertainty in the decision making strategy. Size of the stochastic problem is given in Table 6. All programs have been solved using CPLEX 10.1.1 under GAMS on an $\mathrm{x} 86 / \mathrm{MS}$ Windows with two processors clocking at $2.53 \mathrm{GHz}$ and $4 \mathrm{~GB}$ RAM.

\section{Strategy 1: Risk neutral strategy}

In a risk neutral strategy, the ultimate goal of a

Table 5. Risk aversion coefficients based on fuzzy membership function.

\begin{tabular}{cccccccc}
\hline Hour & $\boldsymbol{\rho}_{\boldsymbol{t}}(\boldsymbol{i})$ & Hour & $\boldsymbol{\rho}_{\boldsymbol{t}}(\boldsymbol{i})$ & Hour & $\boldsymbol{\rho}_{\boldsymbol{t}}(\boldsymbol{i})$ & Hour & $\boldsymbol{\rho}_{\boldsymbol{t}}(\boldsymbol{i})$ \\
\hline 1 & 0.54 & 7 & 0.54 & 13 & 0.16 & 19 & 0.14 \\
2 & 0.74 & 8 & 0.47 & 14 & 0.20 & 20 & 0.00 \\
3 & 0.95 & 9 & 0.42 & 15 & 0.21 & 21 & 0.05 \\
4 & 1.00 & 10 & 0.30 & 16 & 0.18 & 22 & 0.15 \\
5 & 0.92 & 11 & 0.21 & 17 & 0.16 & 23 & 0.28 \\
6 & 0.79 & 12 & 0.18 & 18 & 0.16 & 24 & 0.58 \\
\hline
\end{tabular}


Table 6. Computational size of the stochastic model.

\begin{tabular}{lcc}
\hline \multicolumn{1}{c}{ Item } & Number \\
\hline Continuous variables & $N_{\omega} N_{T} N_{I} N_{m}+N_{\omega} N_{T} N_{I}+3 N_{T} N_{I}+N_{\omega}+1$ & 115440 \\
Binary variables & $3 N_{T} N_{I}+N_{T} N_{I} N_{k}$ & 660 \\
Constraints & $12 N_{T} N_{I}+5 N_{\omega} N_{T} N_{I}+2 N_{I}+2 N_{\omega}$ & 127680 \\
\hline
\end{tabular}

Table 7. Risk neutral strategy.

\begin{tabular}{cccccc}
\hline Case & $\begin{array}{c}\text { Expected profit } \\
(\$)\end{array}$ & $\begin{array}{c}\text { TCVaR } \\
(\$)\end{array}$ & $\begin{array}{c}\mathbf{H C V a R}_{\text {worst }} \\
(\$)\end{array}$ & $\begin{array}{c}\mathbf{H C V a R}_{\text {best }} \\
(\$)\end{array}$ & $\begin{array}{c}\mathbf{H C V a R}_{\text {mean }} \\
(\$)\end{array}$ \\
\hline A & 14444 & -2245 & -3656 & -338 & -1601 \\
$\mathrm{~B}$ & 16820 & -4911 & -5524 & -1103 & -3007 \\
$\mathrm{C}$ & 29241 & 12752 & -2742 & -337 & -1006 \\
$\mathrm{D}^{*}$ & 31617 & 10388 & -4151 & -1045 & -2442 \\
\hline
\end{tabular}

*Solution time of Case D: $55 \mathrm{sec}$

Table 8. Long-term risk management strategy.

\begin{tabular}{cccccc}
\hline Case & $\begin{array}{c}\text { Expected profit } \\
(\$)\end{array}$ & $\begin{array}{c}\text { TCVaR } \\
(\$)\end{array}$ & $\begin{array}{c}\mathbf{H C V a R}_{\text {worst }} \\
(\$)\end{array}$ & $\begin{array}{c}\mathbf{H C V a R}_{\text {best }} \\
(\$)\end{array}$ & $\begin{array}{c}\text { HCVaR }_{\text {mean }} \\
(\$)\end{array}$ \\
\hline $\mathrm{A}$ & 14444 & -763 & -3655 & 0 & -1601 \\
$\mathrm{~B}$ & 16028 & -1992 & -3732 & -337 & -2522 \\
$\mathrm{C}$ & 29243 & 14628 & -2742 & 0 & -1005 \\
$\mathrm{D}^{*}$ & 29283 & 15434 & -2302 & 0 & -872 \\
\hline
\end{tabular}

* Solution time of Case D: 2 min $18 \mathrm{sec}$

GENCO is to maximize the profit of participation in electricity market. Therefore, it is assumed that in Eq. (3), $\alpha=0$ and Risk Constraint (54) is ignored. The resulting profit and corresponding risk by applying this strategy are indicated in Table 7 . Risk assessment is carried out by calculating longterm CVaR, which corresponds to the whole planning horizon (TCVaR), and short-term CVaRs, which are measured on an hourly basis (HCVaRs). In this sense, $\mathrm{HCVaR}_{\text {worst }}$ and $\mathrm{HCVaR}_{\text {best }}$ represent the worst and the best amounts of short-term CVaR in the planning horizon, respectively, and $\mathrm{HCVaR}_{\text {mean }}$ is defined as the average $\mathrm{HCVaR}$ of 24 hours. For instance, in Case D, the worst HCVaR and the best HCVaR in the scheduling day are $-\$ 4151$ and $-\$ 1045$, respectively, which means that even in the best situation, the expected value of the $5 \%$ lowest profits of the GENCO for an hour is a negative value that shows a great loss. It is worth mentioning that adopting risk neutral strategy provides relatively greater profits than the methods with risk management tools. However, the GENCO is exposed to high level of risk as it will be shown in the following.

\section{Strategy 2: Long-term risk management}

Self-scheduling of the GENCO for participating in different markets is carried out considering long-term risk management technique. It is assumed that the short-term risk is ignored by the decision maker. Consequently, risk measure part of the objective function (3) is substituted by maximizing long-term CVaR and TCVaR, defined by Eq. (53). A weighting factor $\left(\alpha_{L}\right)$ is utilized in order to make desirable trade-off between risk and profit. The amount of $\alpha_{L}$ is adjusted according to our proposed fuzzy approach when the average price of the day has been used to choose the appropriate value for the linear fuzzy membership function. Table 8 shows the result of applying this method when $\alpha_{L}=0.34$. As a result of diversification in the portfolio selection, the more the investment options provided, the less the long-term risk is. However, an exception can be seen when the possibility of involvement in forward contracts is added. In this case, risk lover GENCO has chosen to benefit from arbitrage opportunities that are provided through purchasing power from forward contracts and sells power in the pool market; consequently, the risk has increased.

\section{Strategy 3: Proposed portfolio selection method with long-term and short-term risk management \\ In this part, two examples are provided for making} comparison between the proposed portfolio selection approach and the two aforementioned strategies. 
Table 9. Proposed portfolio selection strategy (Example 1).

\begin{tabular}{cccccc}
\hline Case & $\begin{array}{c}\text { Expected profit } \\
(\$)\end{array}$ & $\begin{array}{c}\text { TCVaR } \\
(\$)\end{array}$ & $\begin{array}{c}\mathbf{H C V a R}_{\text {worst }} \\
(\$)\end{array}$ & $\begin{array}{c}\mathbf{H C V a R}_{\text {best }} \\
(\$)\end{array}$ & $\begin{array}{c}\mathbf{H C V a R}_{\text {mean }} \\
(\$)\end{array}$ \\
\hline $\mathrm{A}$ & 13171 & 0 & -2679 & 0 & -873.5 \\
$\mathrm{~B}$ & 13691 & 0 & -2437 & 0 & -866 \\
$\mathrm{C}$ & 28806 & 12752 & -1358 & 182 & -418 \\
$\mathrm{D}^{*}$ & 28338 & 10388 & -985 & 1077 & -77 \\
\hline
\end{tabular}

* Solution time of Case D: 3 mins $31 \mathrm{sec}$

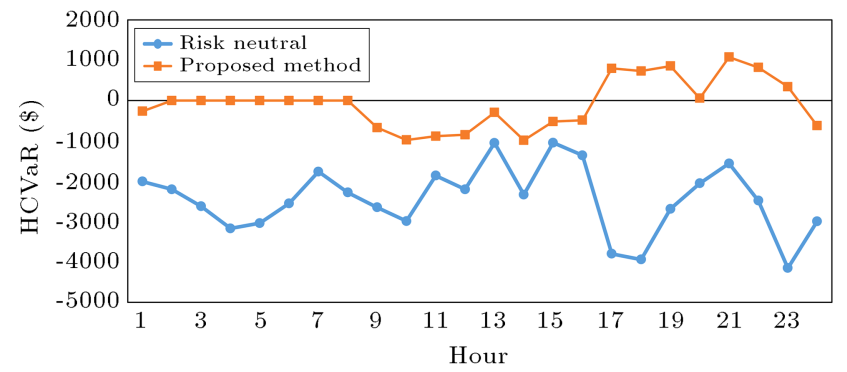

Figure 5. HCVaRs of the proposed method and risk neutral portfolio selection strategy (Case D).

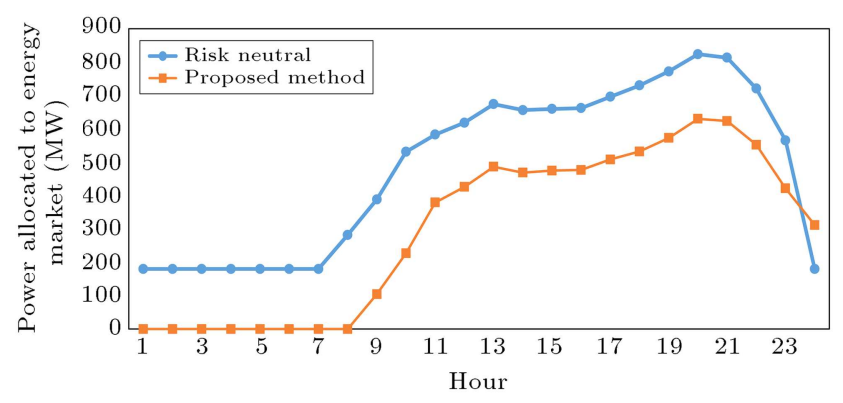

Figure 6. Power allocated to energy market (Case D).

Example 1: In the first example, the resulting TC$\mathrm{VaR}$ in the risk neutral strategy is considered as the minimum accepted value for long-term CVaR of the GENCO, $\Omega_{L}$. In Cases A and B, for which risk neutral strategy results in a negative $\mathrm{TCVaR}, \Omega_{L}$ is set to zero. Table 9 shows the results of this example.

By comparing the expected profits of Case D in Tables 7 and 9 , a total profit of $\$ 31617$ is obtained with risk neutral strategy and $\$ 28338$ by applying the proposed method, which is $10.3 \%$ lower. Instead, adopting the proposed portfolio selection strategy has decreased short-term risk so that $\mathrm{HCVaR}_{\text {worst }}$, $\mathrm{HCVaR}_{\text {best }}$, and $\mathrm{HCVaR}_{\text {mean }}$ have significantly been improved by $76.2 \%, 203 \%$, and $96.8 \%$, respectively. Figure 5 shows the resulting HCVaR by applying the proposed methodology and risk neutral portfolio selection approach. Since Case D is the most comprehensive case, optimal amount of power, which is allocated to each market in this case, is depicted in Figures 6-8. Figure 6 shows the power allocated to energy market with which risk neutral and the proposed method

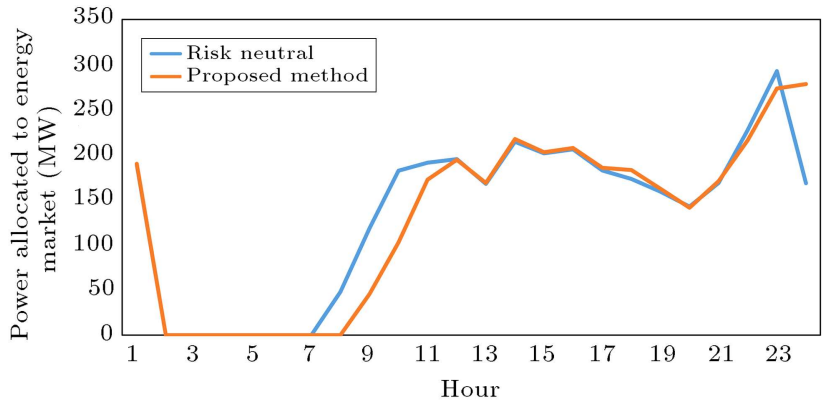

Figure 7. Power allocated to ancillary services markets (Case D).

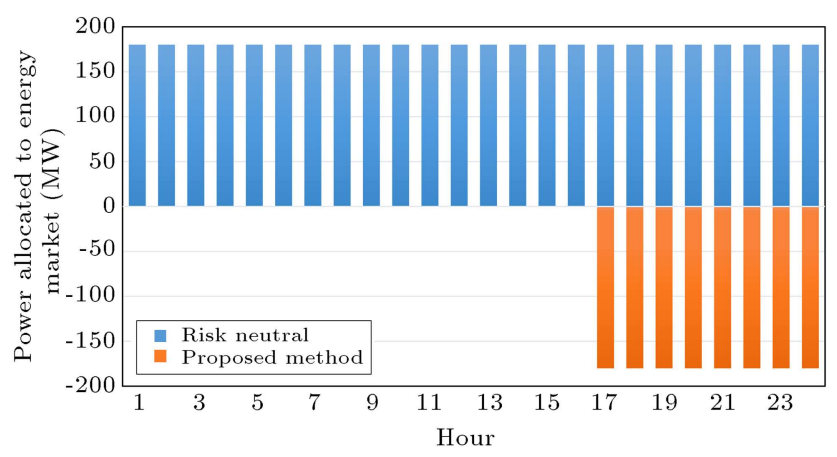

Figure 8. Power allocated to forward contracts (Case D).

have been applied. Furthermore, the expected energy price is depicted in this figure. It should be noted that in risk neutral strategy, the GENCO purchases maximum allowed power through forward contracts and sells it in pool market in hope of gaining more profit in spite of being exposed to higher amount of risk. Figures 7 and 8 show the power allocated to ancillary services markets and forward contracts, respectively. In Figure 8 positive/negative values correspond to buying/selling power through forward contracts.

Example 2: In this example, the limitation of longterm risk is considered equal to the TCVaR value obtained by long-term risk management strategy. Consequently, when the proposed method and long-term risk management strategy provide the same amount of TCVaR, a comparison between the expected profits and short-term risk of these two strategies can be 
Table 10. Proposed portfolio selection strategy (Example 2).

\begin{tabular}{cccccc}
\hline Case & $\begin{array}{c}\text { Expected profit } \\
(\$)\end{array}$ & $\begin{array}{c}\text { TCVaR } \\
(\$)\end{array}$ & $\begin{array}{c}\text { HCVaR }_{\text {worst }} \\
(\$)\end{array}$ & $\begin{array}{c}\text { HCVaR }_{\text {best }} \\
(\$)\end{array}$ & $\begin{array}{c}\text { HCVaR }_{\text {mean }} \\
(\$)\end{array}$ \\
\hline A & 13948 & -763 & -2679 & 0 & -984 \\
B & 13691 & -1992 & -2437 & 0 & -866 \\
C & 28817 & 14628 & -1358 & 140 & -423 \\
D $^{*}$ & 28338 & 15434 & -985 & 1077 & -423 \\
\hline
\end{tabular}

* Solution time of Case D: 3 mins $33 \mathrm{sec}$

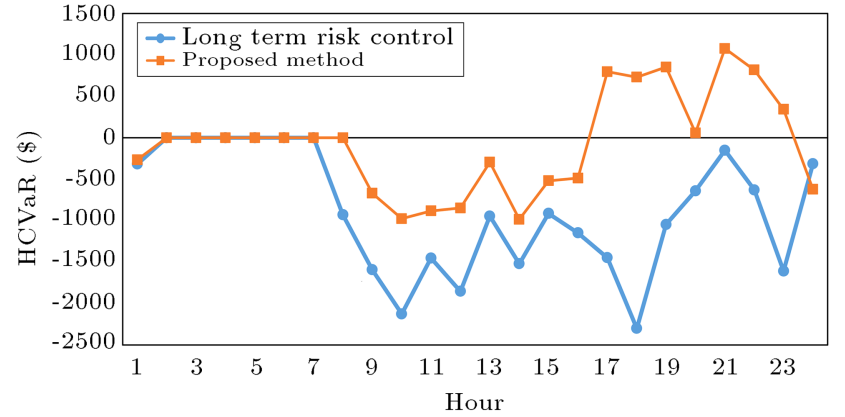

Figure 9. HCVaRs of the proposed method and long-term risk management strategy (Case D).

made. The results of this example are shown in Table 10. Furthermore, Figure 9 depicts the resulting HCVaR by applying the proposed methodology and long-term risk management approach. By comparing Tables 8 and 10 , it can be concluded that with the same TCVaR value, the proposed risk management technique improves the $\mathrm{HCVaR}_{\text {mean }}$ of the scheduling day by $51.4 \%$, while the expected profit is only reduced by $3.34 \%$. This is a remarkable advantage that clearly illustrates the effectiveness of the proposed method. From the viewpoint of a decision maker, it is a merit that the short-term risk is significantly decreased with a low cost and the desired long-term risk preferences are satisfied as well.

\section{Conclusions}

This paper provides suppliers with a novel decisionmaking tool that accounts for both long-term and short-term risk aversion preferences of the GENCO. Considering the uncertainty in electricity market price as the main source of risk, optimal strategies to participate in forward contracts, and energy and ancillary services markets were devised. Because of the imprecise nature of the decision maker's judgment, appropriate modelling of risk aversion attitude of the GENCO is another challenge. This paper used fuzzy satisfaction theory to express decision maker's attitude toward risk. By applying the proposed method, not only trading loss over the whole scheduling horizon could be controlled, but also the amount of imposed loss during every time period could be reduced. Precise modelling of the production units by considering prevailing operational constraints of the thermal units and utilizing the advanced CPLEX solver under GAMS for solving this stochastic mixed-integer programming problem make the developed algorithm suitable for practical operation.

The advantages of the proposed method were well illustrated by numerical results and comparing three different risk management strategies, namely, risk neutral strategy, long-term risk management method, and the proposed portfolio selection approach, through four attractive cases. According to the obtained results, by applying the proposed method, in addition to meeting the desired long-term risk requirement, the short-term risk can be substantially decreased in expense of a small reduction in the expected profit.

Finally, in this paper, the optimization of portfolio selection from the viewpoint of a generation company was analyzed. In future, it would be interesting to devise an optimal risk management strategy for independent system operator or other electricity market players such as transmission or distribution network providers and customers. Also, this paper focused on stochastic electricity market price as the main source of uncertainty, while behavior of other market participants, operational failure of generation units, unexpected outages of transmission lines, renewable resources production, etc. are also attractive ideas to be explored with this risk assessment strategy.

\section{Nomenclature}

\section{Variables}

$\Phi$

$R E_{t \omega} \quad$ Revenue obtained from energy market in period $t$ and scenario $\omega$

$R S_{t \omega} \quad$ Revenue obtained from ancillary services market in period $t$ and scenario $\omega$

$C_{t \omega} \quad$ Production cost in period $t$ and scenario $\omega$

$P_{t \omega m} \quad$ Power generated in each production block $m$ in period $t$ and scenario $\omega$ 


$S T C_{t}$
$q_{t}$
$P_{c b}^{S}$
$P_{c b}^{B}$
$P_{t \omega}^{q}$
$\eta_{t}^{s h}$
$\eta^{L}$
$R_{t \omega}^{A S}$
$R_{t \omega}^{x}$

Time varying start-up cost

Dummy variable for computing

start-up cost

Power sold through block $b$ of forward

contract $c$

Power bought in block $b$ of forward

contract $c$

Output power of unit $i$ in period $t$ and scenario $\omega$

$\eta_{t}^{s h} \quad$ The GENCO's VaR in each time period $t$

The GENCO's VaR of the total scheduling horizon

Total power allocated to different ancillary services markets in period $t$ and scenario $\omega$

$R_{t \omega}^{x} \quad$ The amount of power allocated to AGC, spinning reserve, and nonspinning reserve when unit $i$ is on and to non-spinning reserve when it is off; $x=\{A, S, N U, N D\}$,

$P_{t \omega}^{E} \quad$ Power allocated to the energy market

$T C_{t} \quad$ Shutdown time counter

\section{Binary variables}

$A G C_{t} \quad$ Indicator of involvement in AGC market in period $t$

$w_{t} \quad$ Start-up indicator at time period $t$

$U_{t} \quad$ Unit status indicator, which equals 1 if unit $i$ is on at time $t$ and 0 otherwise

$z_{t} \quad$ Shutdown indicator at time period $t$

$U_{c S} \quad$ Indicator of selling through bilateral

$U_{c B} \quad$ Indicator of buying by bilateral contract $c$

$v_{t k} \quad$ Indicator of start-up at hour $t$ and interval $k$

\section{Stochastic variables}

$\lambda_{t \omega}^{E}$

Price of energy market in period $t$ and scenario $\omega$

$\lambda_{t \omega}^{q}$

$\mu_{t}^{s h}, \mu^{L}$

$q=A, S, N$ stands for price of AGC, spinning reserve, and non-spinning reserve markets

$P_{t \omega}^{D}$

Auxiliary variables used to compute the short-term and long-term CVaRs

\section{Constants}

$\pi_{\omega}$

Own load in period $t$ and scenario $\omega$

$S_{L m}$
Probability of occurrence of scenario $\omega$ Slope of segment $m$ in linearized cost function
A

$S C$

$S T_{k}$

$\beta^{s h}, \beta^{L}$

$\alpha$

$R U, R D$

$S U, S D$

$U T$

$T U_{0}$

$M U, M D$

$D T$

$T C_{0}$

$P_{\min }, P_{\max }$

$P_{c}^{S, \max }, P_{c}^{B, \max }$

$R_{\max }^{A}, R_{\max }^{S}$

$R_{\max }^{N}$

$\lambda_{c b}^{S}, \lambda_{c b}^{B}$

$L_{c}$

AL

$S L_{m}$

$\Omega_{L 0}$

$\lambda_{t}^{\max }, \lambda_{t}^{\min }$

$\rho_{t}(i)$

\section{Numbers}

$N_{\omega}, N_{T}, N_{I}$
Generation cost at the minimum output power

Shutdown cost

Cost of the $k$ th interval of start-up cost function

Confidence level used to compute the short-term and long-term CVaRs

Weighting factor to achieve tradeoff between profit and risk

Ramp-up limit and ramp-down limit

Start-up ramp limit and shutdown ramp limit

Number of hours that a unit needs to remain on

Number of hours that a unit has been on at the beginning of scheduling period

Minimum up-time and minimum down-time

Number of hours that a unit needs to remain down

Number of hours that a unit has been off at the beginning of scheduling period

Minimum and maximum output power Maximum power that can be sold and purchased through bilateral contract $c$

Maximum capability for participating in $\mathrm{AGC}$ and spinning reserve markets

Maximum capability for participating in non-spinning reserve market

Selling and purchasing prices of block $b$ in bilateral contract $c$

Time duration of each forward contract (h)

Regulating limit for participating in AGC market

Slope of power block $m$ in piecewise linearized production cost curve

Minimum accepted value for long-term CVaR

Maximum and minimum specified prices greater and lower than prices to which minimum and maximum risk aversion coefficients are assigned

Risk aversion coefficient of unit $i$ in period $t$

Numbers of scenarios, periods, and units, respectively 
$N C^{S}, N C^{B} \quad$ Number of available bilateral contracts

$N b^{S}, N b^{B} \quad$ Numbers of available selling and buying blocks in bilateral contracts

$N_{k}$ Number of intervals of start-up cost

$N_{m}$ Number of blocks in piecewise linearized production cost curve

$S T_{k}$ Start-up cost at segment $k$ in the stair-wise start-up curve

\section{References}

1. Krokhmal, P. and Uryasev, S. "Portfolio optimization with conditional value-at-risk objective and constraints", J. Risk, 4, pp. 11-27 (2001).

2. Artzner, P., Delbaen, F., Eber, J.M., Heath, D., and $\mathrm{Ku}$, H. Multiperiod Risk and Coherent Multi-period Risk Measurement, E.T.H.Zürich, Preprint (2002).

3. Bodie, Z., Kane, A., and Marcus, A.J., Investment, Chicago, Irwin/McGraw-Hill, 4th Edn. (1999).

4. Ni, E., Luh, P.B., and Rourke, S. "Optimal integrated generation bidding \& scheduling with risk management under a deregulated power market", IEEE Transactions on Power Systems, 19, pp. 600-609 (2004).

5. Yamin, H.Y. and Shahidehpour, S.M. "Risk and profit in self-scheduling for gencos", IEEE Transactions on Power Systems, 19, pp. 2104-2106 (2004).

6. Liu, M. and Wu, F.F. "Managing price risk in a multimarket environment", IEEE Transactions on Power Systems, 21, pp. 1512-1519 (2006).

7. Jain, A.K., Srivastava, S.C., Sing, S.N., and Srivastava, L. "Strategic bidding in transmission constrained electricity markets using artificial bee colony algorithm", Electric Power Components and Systems, 40, pp. $1768-1788$ (2012).

8. Luo, X., Chung, C., Yang, H., and Tong, X. "Optimal bidding strategy for generation companies under CVaR constraint", International Transactions on Electrical Energy Systems, 24, pp. 1369-1384 (2014).

9. Garces, L.P. and Conejo, A.J. "Weekly self-scheduling, forward contracting, and offering strategy for a producer", IEEE Transactions on Power Systems, 25, pp. 657-666 (2010).

10. Liu, H. and Hou, Y. "The mean-wcvar based model for ldc's optimal portfolio in multi-energy markets", International Transactions on Electrical Energy Systems, 22, pp. 367-377 (2012)

11. Conejo, A.J., García-Bertrand, R., Carrión, M., Caballero, A., and de Andrés, A. "Optimal involvement in futures markets of a power producer", IEEE Transactions on Power Systems, 23, pp. 703-711 (2008).

12. Xu, Z., Hu, Z., Song, Y., and Wang, J. "Risk-averse optimal bidding strategy for demandside resource aggregators in day-ahead electricity markets under uncertainty", IEEE Transactions on Smart Grid, 8, pp. 96-105 (2017).

13. Rahmani Dabbagh, S. and Sheikh-El-Eslami, M.K. "Risk assessment of virtual power plants offering in energy and reserve markets", IEEE Transactions on Power Systems, 31, pp. 3572-3582 (2016).

14. Artzner, P., Delbaen, F., Eber, J.M., Heath, D., and $\mathrm{Ku}, \mathrm{H}$. "Coherent multi-period risk adjusted values and Bellman's principle", Annals of Operation Research, 152, pp. 5-22 (2007).

15. Gröwe-Kuska, J.N., Heitsch, H., and Römisch, W. "Scenario reduction and scenario tree construction for power management problems", Proc, IEEE Power Technology Conference, 3, pp. 23-26 (2003).

16. Birge, R. and Louveaux, F., Introduction to Stochastic Programming, New York: Springer (1997).

17. Xi, L., Yu, T., Yang, B., and Zhang, X. "A novel multi-agent decentralized win or learn fast policy hillclimbing with eligibility trace algorithm for smart generation control of interconnected complex power grids", Energy Conversion and Management, 103, pp. 82-93 (2015).

18. Xi, L., Yu, T., Yang, B., Zhang, X., and Qiu, X. "A wolf pack hunting strategy based virtual tribes control for automatic generation control of smart grid", Applied Energy, 178, pp. 198-211 (2016).

19. Xi, L., Zhang, Z., Yang, B., Huang, L., and Yu, T. "Wolf pack hunting strategy for automatic generation control of an islanding smart distribution network", Energy Conversion and Management, 122, pp. 10-24 (2016).

20. Li, Z. and Shahidehpour, S.M. "Security-constrained unit commitment for simultaneous clearing of energy and ancillary services markets", IEEE Trans. Power Syst., 20(2), pp. 1079-1088 (2005).

21. "European energy exchange", [Online], Available: http://www.eex.com (2007)

22. "Iberian electricity market operator", [Online], Available: http://www.omip.pt

23. Li, T. and Shahidehpour, S.M. "Price-based unit commitment: a case of Lagrangian relaxation versus mixed integer programming", IEEE Transactions on Power Systems, 20, pp. 2015-2025 (2005).

24. Arroyo, J.M. and Conejo, A.J. "Optimal response of a thermal unit to an electricity spot market", IEEE Trans. Power Syst., 15(3), pp. 1098-1104 (2000).

25. Karimi, M., Shayanfar, H.A., Aghaei, J., and Ahmadi, A. "Mixed integer programming of SecurityConstrained Daily Hydrothermal Generation Scheduling (SCDHGS)", Scientia Iranica, Transactions D., 20(6), pp. 2036-2050 (2013).

26. Friend, I. and Blume, M.E. "The demand for risky assets", American Economics Review, 65, pp. 900-922 (1975). 


\section{Biographies}

Somayyeh Bazmohammadi received the BSc and MSc degrees in Power Engineering from Semnan University, Iran, in 2008 and 2011, respectively. Currently, she is in R\&D Department of Khorasan Regional Electric Company. Her research interests include power system planning, operation, and restructuring.

Asghar Akbari Foroud received the BSc degree in Power Engineering from Tehran University and the MSc and PhD degrees from Tarbiat Modares Univer- sity, Tehran, Iran, in 1997 and 2006, respectively. He is now with Semnan University. His research interests include power system planning, dynamic \& operation, and restructuring.

Najmeh Bazmohammadi received the BSc and MSc degrees in Electrical and Control Engineering from Ferdowsi University of Mashhad, Iran, in 2009 and 2012 , respectively. She is currently PhD student in K. N. Toosi University of Technology, Tehran, Iran. Her research interests include control of large-scale systems, distributed-model predictive control, dynamic decision making under uncertainty, and microgrids. 